Conclusions Cardiac fibrosis, hypertrophy and diastolic dysfunction are enhanced in miR-214 KO animals. The mechanisms of this observation are not caused by mir214 effects in cardiac fibroblasts, but through other mechanisms that need to be further addressed.

\section{THE EFFECT OF INTERLEUKIN 1 ON VASCULAR ANGIOPOIETIN 1 SIGNALLING}

Jay Bilimoria*, Harprit Singh. De Montfort University

10.1136/heartjnl-2017-311726.190

Introduction Angiopoietin 1 (Ang1) is a growth factor that plays a crucial role in maintaining normal vascular function. The main role of Ang1 is to maintain vessels in quiescent, inhibit vascular inflammation and maintain endothelial survival. Ang1 exerts its protective effect by activating Tie2 receptors, which are predominantly expressed on endothelial cells. The ratio between Tie 2 and its co-receptor, Tie1, is a regulatory factor in Ang1 signalling with high levels of Tie1 capable of reducing Ang1-induced Tie2 activation. The activity of Ang1 is aided by Tie2 phosphorylation followed by activation of cascade of downstream signalling pathways including Phosphatidylinositol 3-kinase (PI3Kinase)/AKT and Erk1/2. Interleukin 1 Beta (IL1 $\beta$ ) is a proinflammatory cytokine that acts on microvasculature and large vessels and has been implicated in a range of vascular pathologies including vascular inflammation and atherosclerosis. Various pathophysiological mediators including VEGF and TNF alpha have shown to affect the levels of Tie receptor expression and subsequently influencing Ang1 signalling in endothelial cells; however the involvement of Interleukin $1 \hat{\mathrm{I}}^{2}$ on this pathway has not been reported.

Aim To examine the impact IL1 $\beta$ has on Tie2:Tie1 expression ratio and Angiopoietin1 signalling in endothelial cells.

Method Primary Human umbilical vein endothelial cells (HUVEC) were stimulated with $25 \mathrm{ng} / \mathrm{ml}$ of IL1 $\beta$ at different time periods in the presence or absence of $300 \mathrm{ng} / \mathrm{ml}$ of human recombinant Ang1. Cell lysates from the treated cells were then subjected to immunoprecipitation and Western blotting to analyse Tie receptor levels and signalling molecules associated with Ang1 cellular transduction including PhosphoTie2 (pTie2), and phospho-AKT (pAKT).The levels of target proteins were compared between reactions by quantifying mean intensity of bands. Data is presented as means and SEM of three independent experiments. Statistical significance represented with $\mathrm{p}<0.05$ using Students t test.

Results The expression of Tie 2 and Tie1 protein in endothelial cells treated with IL1 $\beta$ was significantly altered with different Tie2:Tie1 ratio patterns observed at 1 hour and 24 hour. These changes to the Tie2:Tie1 expression patterns also altered the ability of Ang1 to induce Tie2 phosphorylation and downstream signalling molecules including pAKT.

Conclusion This study demonstrates for the first time that IL1 $\beta$ is capable of altering Tie2:Tie1 ratio that subsequently leads to the regulation of Ang1 signalling. These findings provide further insight on how the Angiopoietin1 signalling pathway adapts to the acute and chronic effect of IL1 $\beta$ and how this signalling pathway is important in limiting pro-inflammatory responses of the vasculature.

\section{THE ROLE OF THE PI3K-ALPHA ISOFORM IN CARDIOPROTECTION}

Xavier Rossello*, David He, Jaime A Riquelme, Sean Davidson, Derek M Yellon. The Hatter Cardiovascular Institute, University College London

\subsection{6/heartinl-2017-311726.191}

Introduction Ischaemic preconditioning (IPC) limits infarct size (IS) through the activation of the PI3K-AKT signal cascade, known as the RISK pathway. The identification of the roles of individual PI3K isoforms should aid in the development of isoform-specific agents with improved cardioprotection. We aimed to elucidate the role of the PI3K $\alpha$ isoform in cardioprotection.

Methods Male C57BL/6 mice were randomly allocated between groups in isolated perfused Langendorff experiments subjected to $35 \mathrm{~min}$ ischaemia and 2 hour reperfusion. Two distinct PI3K $\alpha$-isoform specific inhibitors $(3 \mu \mathrm{M}$ of $\mathrm{G} 326$ and BYL719) were administered during the IPC protocol (4-cycles of $5 \mathrm{~min}$ ischaemia and $5 \mathrm{~min}$ reperfusion), or at reperfusion. PI3K activity was measured by Western blot analyses of AKT phosphorylation. Insulin was used as $\mathrm{PI} 3 \mathrm{~K} \alpha$ canonical activator. In addition, these studies were also undertaken using an in vivomouse model. Finally, expression of PI $3 \mathrm{~K} \alpha$ protein levels was evaluated by Western blot in whole mouse heart and human atrial tissues (REC No. 00/0275), as well as in primary adult ventricular mouse cardiomyocytes and mouse cardiac endothelial cell (MCEC) line.

Results In the isolated Langendorff-perfused model, IPC reduced IS compared to control (49 $\pm 4 \%$ vs $23 \pm 2 \%$, $\mathrm{p}<0.001)$. Protection was not abolished using either G326 (26 $\pm 3 \%)$ or BYL719 $(25 \pm 3 \%)$ when administered during IPC (Figure 1A) despite the phosphorylation of AKT being inhibited. Interestingly, these same isoform inhibitors did block pro-

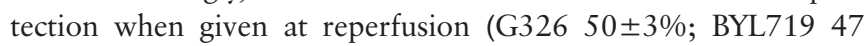
$\pm 4 \%$ ) (Figure $1 \mathrm{~B}$ ), in addition to blocking the AKT phosphorylation revealing a distinct role for the PI $3 \mathrm{~K} \alpha$ isoform during the early moments of reperfusion. Neither drug affected IS on its own. In support, the canonical PI $3 \mathrm{~K} \alpha$ activator, insulin, when given at reperfusion reduced myocardial IS compared to control $(25 \pm 2$ vs $55 \pm 4 \%, \mathrm{p}<0.001)$ and this protection was abolished by G326 (Figure 2A). These experiments were confirmed in vivo when using the $\mathrm{PI} 3 \mathrm{~K} \alpha$ isoform inhibitor at reperfusion to block IPC protection (Figure 2B). On a tissue level, PI $3 \mathrm{~K} \alpha$ is expressed at the same level in mouse heart and human atrium. Interestingly, PI3K $\alpha$ levels in MCEC were about 3-fold higher compared to adult ventricular mouse cardiomyocytes $(\mathrm{p}=0.047)$.

Conclusions Using a targeted pharmacological approach, we have demonstrated that the PI3KII \pm isoform is required during the reperfusion phase for protection conferred by IPC. The translational perspective is highlighted by the observation that human heart tissue expresses PI3KÎ \pm . This suggests that strategies specifically enhancing the \pm isoform of PI3 kinase at reperfusion promotes tissue salvage and, as such, could provide an attractive target for clinical treatment of ischaemiareperfusion injury. 